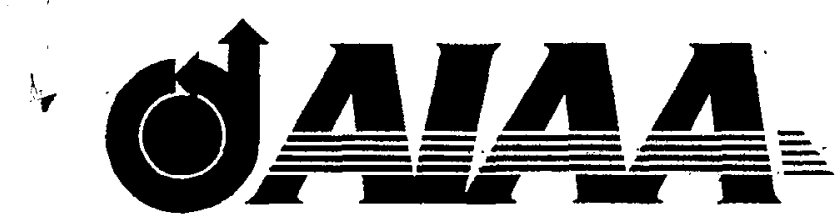

\title{
AIAA 2000-0433
}

\section{Coupling Between Fluid Dynamics and Combustion in a Laminar Vortex Ring}

Shin-Juh Chen and Werner J.A. Dahm

Laboratory for Turbulence and Combustion (LTC)

Department of Aerospace Engineering

The University of Michigan

Ann Arbor, MI

Grétar Tryggvason

Laboratory for Turbulence and Combustion (LTC)

Department of Mechanical Engineering \& Applied Mechanics

The University of Michigan

Ann Arbor, MI

\section{8th Aerospace Sciences \\ Meeting \& Exhibit \\ 10-13 January 2000 / Reno, NV}




\title{
Coupling Between Fluid Dynamics and Combustion in a Laminar Vortex Ring
}

\author{
Shin-Juh Chen ${ }^{1}$ and Werner J.A. Dahm ${ }^{2}$ \\ Laboratory for Turbulence and Combustion (LTC) \\ Department of Aerospace Engineering \\ The University of Michigan \\ Ann Arbor, MI 48109-2140 \\ Grétar Tryggrvason ${ }^{3}$ \\ Laboratory for Turbulence and Combustion (LTC) \\ Department of Mechanical Engineering \& Applied Mechanics \\ The University of Michigan \\ Ann Arbor, MI 48109-2121
}

\begin{abstract}
Flame-vortex interactions are critical to the understanding of turbulent reacting flows. The impact of exothermicity on reacting vortex rings is investigated both numerically and experimentally to assess the dominant effect of heat release. Experimental observations of ring trajectories show an initial increase in ring speed in the early stage, followed by a large reduction in speed. It is found numerically that dilatation due to combustion heat release is the dominant effect over enhanced diffusivities in a reacting vortex ring. Increasing fuel volume in the ring beyond a critical limit, obtained from a simple model, actually decreases the amount of heat release during the early stage of the interaction. In addition, the increase in ring circulation led to a decrease in ring speed in the early stage of formation. Nitrogen dilution of the propane fuel reduces the flame luminosity and burnout time, as well as changes in details of the formation and dissipation of the luminous cap, with little change in the primary structure or dynamics of the interaction. The numerical simulations were successful in explaining most of the experimental observations, however, differences in flame structure and ring dynamics attributed to radiative heat loss were inconclusive when radiative heat loss was modeled as an overall decrease in flame temperature.
\end{abstract}

\section{INTRODUCTION}

Flame-vortex interactions are considered a central component of turbulent combustion, and it is for this reason that various flamevortex configurations play a key role in combustion science since the initial study of Marble (1985). Exothermicity due to combustion heat release can dramatically alter some of the most fundamental aspects found in turbulent flows. Studies of such flame-vortex configurations are providing important new insights into elementary transport and reaction processes, since these interactions contain many of the fundamental elements of flow, transport and combustion that are present in turbulent flames. Moreover, these elements can be investigated under more controllable conditions than is possible in direct studies of turbulent reacting flows.

The study of diffusion flame-vortex ring interactions can be conducted experimentally in several ways, as depicted in Fig. 1. Strawa and Cantwell (1985), Vandsburger et al (1988), Chen et al (1991), Hsu et al (1993), and Takahashi et al (1996) have studied these interactions by examining vortices generated in

1. Graduate Student; AIAA Student Member.

2. Professor; AIAA Senior Member; Corresponding author. 3. Professor.

Copyright $₫ 2000$ by Shin-Juh Chen pulsed or turbulent jet diffusion flames. In Fig. 1a, some of the fundamental aspects of these interactions are not easily accessible for close study. Recent studies of Rolon et al (1995), and Thévenin et al (1996), and Renard et al (1999) have used the configuration of a counterflow diffusion flame with a vortex ring coming from either the fuel or the oxidizer side as shown in Fig. 1b. In this configuration, the vortex ring is already fully formed as it approaches the diffusion flame, and consists entirely of fuel or oxidizer.

In this paper, the configuration shown in Fig. 1c was used. This configuration has been studied, experimentally under microgravity conditions by Chen \& Dahm (1997, 1998, 1999a,b), and under normal gravity conditions by Park \& Shin (1997) and You et al (1998). The present interaction is similar to that of Karagozian \& Manda (1986) and Manda \& Karagozian (1988), where the ring consists of fuel and oxidizer, and combustion occurs from the start of the ring formation process. This configuration can provide a strong coupling between the vortex ring and diffusion flame. A diffusion flame initially exists at the oxidizer-fuel interfaces near the nozzle exit plane. Fuel is issued impulsively from the exit of the round nozzle and rolls up with oxidizer and the diffusion flame to form a laminar reacting vortex ring. At the same time, heat release due to combustion affects the vortex ring and the transport properties within it. The interaction proceeds until the fuel in the ring has been consumed.

Here we present experimental results for the reacting vortex ring 


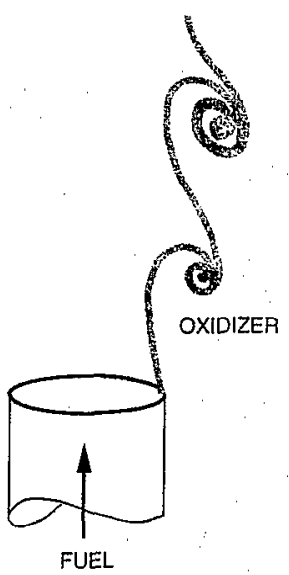

(a)

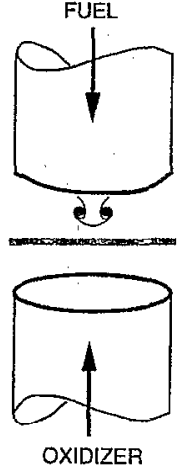

(b)

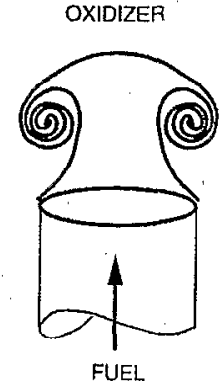

(c)

Figure 1. Configurations for studying flame-vortex interactions. (a) Direct investigation of vortices generated in a turbulent diffusion flame. (b) Counterflow diffusion flame with a vortex ring filled only with fuel and interacting with the diffusion flame. (c) Configuration used in this study with a vortex ring composed of both fuel and oxidizer and wrapping the already formed diffusion flame at the nozzle exit.

in microgravity that reveal certain aspects of heat release effects, as well as numerical simulations that clarify the dominant mechanisms from which these effects originate. Some of experimental results were presented in Chen \& Dahm (1998). The numerical simulations were conducted with the assumptions of unity Lewis and Schmidt numbers, and the Navier-Stokes and mixture fraction equations were solved to reveal the impact of heat release in a vortex ring. The effects of heat release, fuel volume, ring circulation, stoichiometry, and radiative heat loss on the resulting flame structure and dynamics are discussed to shed light on the coupling between flow field and combustion in a reacting laminar vortex ring.

\section{METHODS}

In this study, experiments were conducted under microgravity

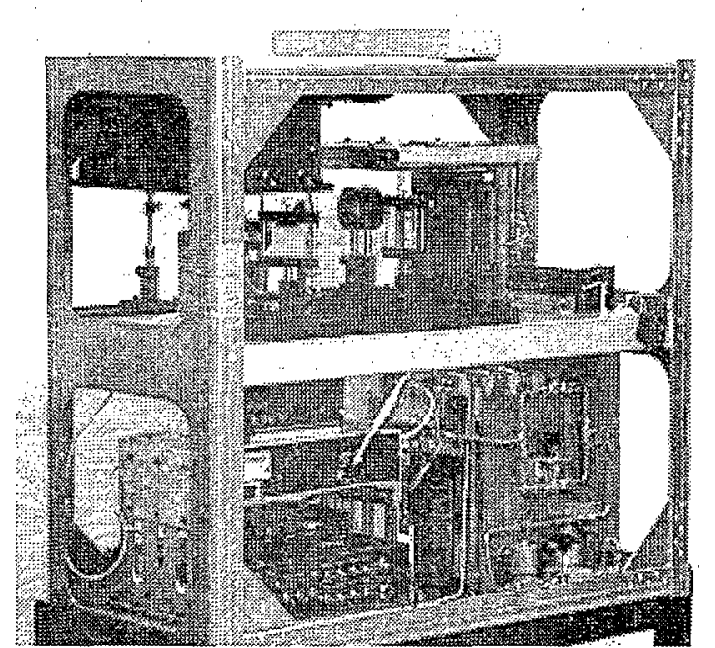

Figure 2. Experiment drop package. conditions at the NASA 2.2 seconds drop tower facility. As shown in Fig. 2, all hardware must be contained inside a drop frame with dimensions of $40.6 \mathrm{~cm} \times 96.5 \mathrm{~cm} \times 83.8 \mathrm{~cm}$ and weight not exceeding $158 \mathrm{~kg}$. Numerical simulations were also conducted to explain some of the experimentally observed phenomena and to assess the dominant effects of combustion heat release in a reacting vortex ring.

\subsection{Microgravity experiments}

The apparatus of the current study was used in the previous studies of Chen \& Dahm $(1997,1998,1999 a, b)$ and is shown in Fig. 2. The top shelf of the drop package contains the $20 \mathrm{~cm} \mathrm{x}$ $20 \mathrm{~cm} \times 25 \mathrm{~cm}$ test section made of clear Lucite, a $640 \times 480$ $\mathrm{CCD}$ array which records the resulting luminosity of a reacting vortex ring with all frames shown in inverse grayscale in this paper, and a spark igniter box for the gencration of a diffusion flame. An axisymmetric circular nozzle and plenum are attached below the top shelf for the generation of vortex rings. The bottom shelf houses the controlling electronics, on-board computer, batteries, pressurized fuel tank, a vacuum tank, flowmetering valves, a fast-acting solenoid valve, and the necessary fittings and tubings.

A vortex ring is generated by impulsively issuing fuel through the circular nozzle of diameter $D=2 \mathrm{~cm}$ into an air environment which is held at atmospheric conditions inside the test section. A schematic of the apparatus is shown in Chen \& Dahm (1998). The experiment is conducted as follows. Initially, there is a closed iris that separates the fuel and air interfaces as shown in Fig. 3. The iris opens prior to the drop and exposes the fuel and

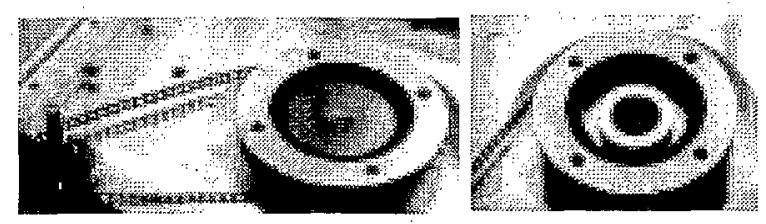

Figure 3. (Left) Closed iris. (Right) Open iris which exposes the circular nozzle and the igniter and allows the formation of a diffusion layer between the fuel and oxidizer interfaces. 


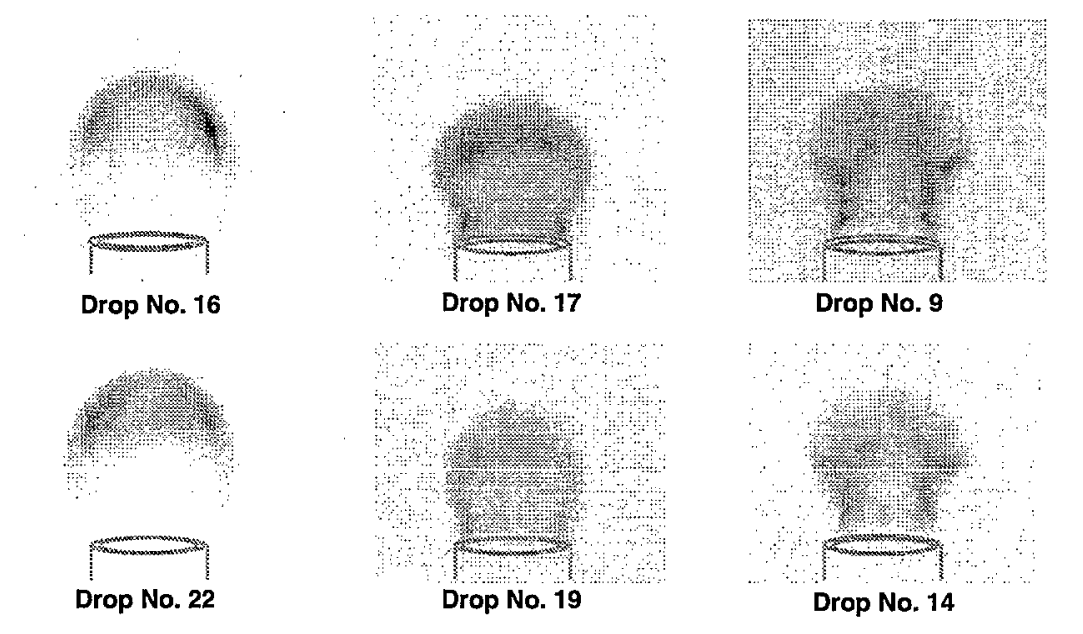

(a)

(b)

(c)

Figure 4. Symmetric and repeatable interactions are accessible under microgravity conditions.

Circulations in $(b)$ and $(c)$ are respectively six times and eleven times that of $(a)$.

air interfaces to form a combustible diffusion layer. Through the opening of a vacuum reservoir, the layer is drawn toward a spark igniter to form a diffusion flame which flattened after a 30-40 $\mathrm{ms}$ of drop time. Immediately after the drop, a solenoid valve opcns and relcascs pressurized fucl into the plenum, and cxits through the circular nozzle to generate a vortex ring that wraps the already formed diffusion flame around itself (Karagozian et al 1988; Southerland et al 1991). The resulting reacting vortex ring moves away from the nozzle and burns until the fuel enclosed in the ring has depleted.

The ring circulation $\Gamma$ and ejected fuel volume $V_{F}$ are determined from hot-wire anemometry measurements of velocity at the center of the nozzle exit plane with the assumption of a top-hat velocity profile. A typical fuel injection velocity programme exhibits a sharp rise when the solenoid valve opens and a slow decay when the valve closes. By varying the fuel tank pressure, solenoid valve timing sequence, and flowmetering valve settings, different ring circulations and fuel volumes can be achieved to obtain a wide range of flame-vortex interactions.

Fuels considered in this study include methane, ethane, propane, and nitrogen diluted fuels reacting with air, and are investigated under various hydrodynamic conditions.

\subsection{Numerical Simulations}

The axisymmetric, viscous, and compressible form of the Navier-Stokes equations were solved for zero gravity on a staggered $66 \times 130$ computational grid that spans the $8 \mathrm{~cm} \times 16$ $\mathrm{cm}$ axisymmetric half-domain. The left boundary is the axisymmetry, and outflow boundaries are on the right and top. The bottom boundary consists of a no-slip wall with a uniform fuel inflow through a $1-\mathrm{cm}$ radius nozzle over a specified duration.

A one-step infinitely fast reaction with unity Lewis and Schmidt numbers was used. This allows the replacement of the species and energy equations with a single equation for the mixture fraction field, $\zeta$. Kinematic viscosity and scalar diffusivity were taken to vary with temperature as $T(\zeta)^{1.7}$, and molecular weight was assumed to be a constant so that density varied as $T(\zeta)^{-1}$.

The advection terms were discretized using the third-order accurate QUICK scheme, and the diffusion terms with a secondorder centered scheme. The time integration was performed by using a first-order explicit scheme. A projection method was used to solve the Navier-Stokes equations, with the pressure field obtained from a Poisson equation by a multigrid method. Dilatation due to combustion heat release was represented by a source term in the Poisson equation, and was expressed in terms of mixture fraction by rearranging the continuity and scalar transport equations along with the ideal gas equation of state (e.g., Baum et al 1990).

The reaction of methane in air was considered in the numerical studies. The stoichiometric mixture fraction and maximum temperature were fixed at the methane-air values of 0.055 and $2226 \mathrm{~K}$, with fuel and ambient temperatures at $300 \mathrm{~K}$.

\section{RESULTS AND DISCUSSION}

The experimental study spans a wide range of hydrodynamic and chemical parameters. For a given nozzle diameter $(2-\mathrm{cm}$ in this study), there is a finite amount of fuel that can be injected into the vortex ring before reaching the overfilled limit. This limit corresponds to length-to-diameter ratio of 3.6-4.4 (Gharib et al 1998) which is based on an equivalent cylindrical volume of fluid emanating from the nozzle, and corresponds to limiting fuel volume of $23-28 \mathrm{~cm}^{3}$. Even though the results from Gharib et al (1998) were for nonreacting vortex rings, the agreement with the current experiment is quite good (Chen \& Dahm, 1999a,b). This study will focus only on non-overfilled cases.

Under microgravity conditions where buoyancy is effectively eliminated, very repeatable and symmetric results can be obtained. Figure 4 shows results from three different cases taken from six separate drops, and the images are time-matched as closely as allowable. It is evident in these figures that highly symmetric and repeatable flame-vortex interactions are 


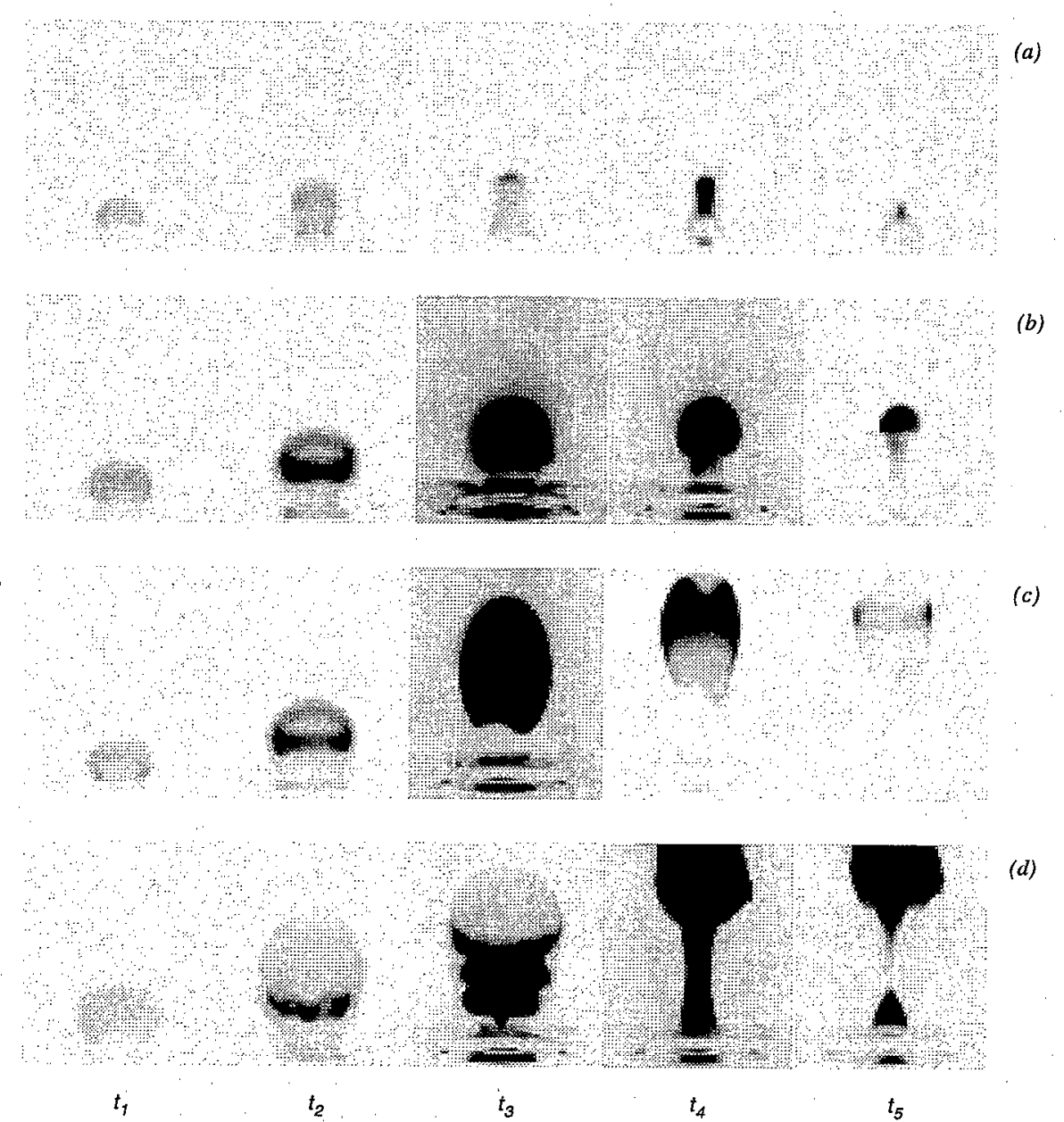

Figure 5. Propane-air combustion for various fuel volume and ring circulation.

(a) $\Gamma=125 \mathrm{~cm}^{2} / \mathrm{s}, V_{F}=4 \mathrm{cc}, t_{1}=0 \mathrm{~ms}, t_{2}=33 \mathrm{~ms}, t_{3}=100 \mathrm{~ms}, t_{4}=133 \mathrm{~ms}, t_{5}=167 \mathrm{~ms}$.

(b) $\Gamma=150 \mathrm{~cm}^{2} / \mathrm{s}, V_{F}=9 \mathrm{cc}, t_{1}=0 \mathrm{~ms}, t_{2}=67 \mathrm{~ms}, t_{3}=167 \mathrm{~ms}, t_{4}=267 \mathrm{~ms}, t_{5}=367 \mathrm{~ms}$.

(c) $\Gamma=150 \mathrm{~cm} 2 / \mathrm{s}, V_{F}=22 \mathrm{cc}, t_{1}=0 \mathrm{~ms}, t_{2}=100 \mathrm{~ms}, t_{3}=533 \mathrm{~ms}, t_{4}=1000 \mathrm{~ms}, t_{5}=1133 \mathrm{~ms}$.

(d) $\Gamma=930 \mathrm{~cm}^{2} / \mathrm{s}, V_{F}=30 \mathrm{cc}, t_{1}=0 \mathrm{~ms}, t_{2}=33 \mathrm{~ms}, t_{3}=67 \mathrm{~ms}, t_{4}=330 \mathrm{~ms}, t_{5}=430 \mathrm{~ms}$.

accessible in the experimental studies.

Figures 5 and 6 suggest that exothermicity due to combustion heat release has a pronounced impact on both the structure and dynamics of reacting vortex rings. The impact on the ring dynamics is clearly seen in Figs. 7 and 8 showing the time history of non-dimensional flame heights for several cases. Moreover, Fig. 7 suggests that fuel volume may also play a role on the resulting ring dynamics. In order to discern the dominan effect of combustion heat release, numerical simulations were conducted; this allow numerical studies that are not accessible in experimental settings to $b c$ conducted and provide access to a wider range of parameters to be investigated than is permissible in experiments, but requires some modeling of certain physical processes.

\subsection{Effect of Heat Release}

Typical flame heights obtained from experiments are shown in Figs. 7 and 8 with the ring height and time hydrodynamically scaled as $H^{*}=H / D$, and $t^{*}=t \Gamma / D^{2}$, where $H$ is the visible flame heights for several different fuels, circulations, and fuel volumes. In the case of nonreacting vortex rings, if the core structure are similar, then one would expect the ring trajectories to collapse to a single curve when plotted in these hydrodynamically scaled variables, however, they do not for the trajectories of reacting vortex rings. This suggests that heat release appears to have an impact on the reacting ring trajectories. For an inviscid ring the resulting constant speed would produce a straight trajectory having a slope that depends weakly on the vortex core size and internal structure. As for a viscous ring, viscous diffusion increases the core size with time and progressively reduces the ring speed similar to the trend seen in Figs. 7 and 8. However, no reasonable core size for a constant viscosity ranging from cold to hot gas values will replicate the experimental observations. Even spatial and temporal variations in viscosity are insufficient to explain the trend in these trajectories. This strongly suggests that a heat release effect other than the simple increase in viscosity appcars to dominate the reacting vortex ring trajectories.

Numerical simulations were conducted for methane-air reaction to examine the origins of these effects. Methane is considered 


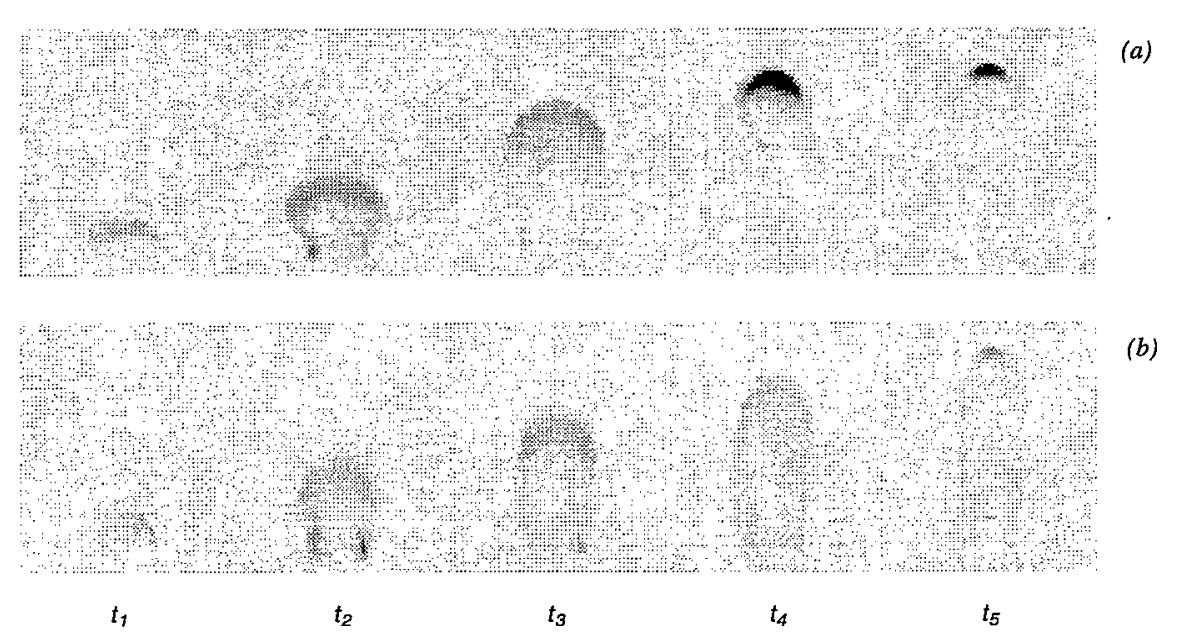

Figure 6. Nitrogen diluted propane-aircombustion. (a) Propane diluted to $40 \%$ with nitrogen, $\Gamma=196 \mathrm{~cm}^{2} / \mathrm{s}$, $V_{F}=24 \propto, t_{1}=0 \mathrm{~ms}, t_{2}=67 \mathrm{~ms}, t_{3}=233 \mathrm{~ms}, t_{4}=367 \mathrm{~ms}, t_{5}=500 \mathrm{~ms}$. (b) Propanc diluted to $20 \%$ with nitrogen, $\Gamma=217 \mathrm{~cm}^{2} / \mathrm{s}, V_{F}=26 \mathrm{cc}, t_{1}=0 \mathrm{~ms}, t_{2}=67 \mathrm{~ms}, t_{3}=133 \mathrm{~ms}, t_{4}=200 \mathrm{~ms}, t_{5}=300 \mathrm{~ms}$.

here in order to remove the effect of radiative heat loss due to the presence of soot; this subject is discussed below. The relative importance of heat release effects on diffusion and dilatation due to reaction and heat conduction in the fluid are compared in Fig. 9. The mixture fraction fields indicate that there are differences between the nonreacting ring and the reacting ring with dilatation turned off. These differences are mainly enhanced molecular diffusion which is responsible for the more diffused scalar field, and the decrease in ring speed in the later stage of interaction due to enhanced viscous diffusion in the reacting case with dilatation turned off. However, the largest differences are seen when comparing to the reacting case with dilatation turned on, where there is a large reduction in ring speed, and the scalar field has considerably spread radially apart. These sharp differences are therefore attributable to volumetric expansion due to combustion heat release.

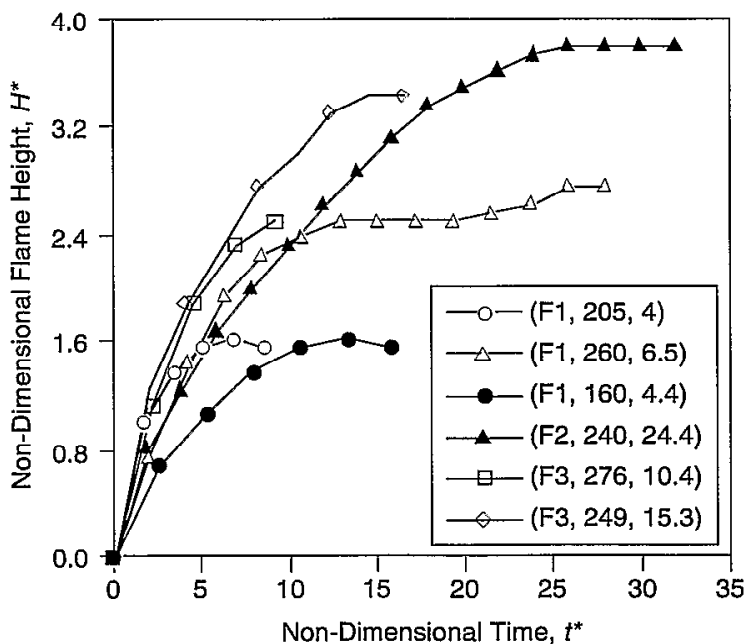

Figure 7. Typical trajectories of reacting vortex rings from experiments under microgravity. Legend gives fuel $\left(\mathrm{F}_{1}=100 \% \mathrm{C}_{3} \mathrm{H}_{8}, \mathrm{~F}_{2}=\right.$ $40 \% \mathrm{C}_{3} \mathrm{H}_{8}+60 \% \mathrm{~N}_{2}$, and $\mathrm{F}_{3}=20 \% \mathrm{C}_{3} \mathrm{H}_{8}+80 \% \mathrm{~N}_{2}$ ), circulation $\Gamma$ $\left(\mathrm{cm}^{2} / \mathrm{s}\right)$, and fuel volume $V_{F}\left(\mathrm{~cm}^{3}\right)$.
By examining the trajectories of these three cases, Fig. 10 clearly shows that dilatation is the duminant effect over enhanced diffusivities when combustion heat release is present in methaneair reaction. There are no differences in the trajectories of nonreacting case and reacting case with dilatation turned off during the early stage of interaction, that is for $t^{*}<8$. Dilatation is responsiblc for the incrcase in ring speed in the early stage, and the sharp reduction in ring speed in the later stage for the reacting case with dilatation turned on. This is consistent with the experimentally observed reduction in ring speed over time in Figs. 7 and 8, and with the numerical results in Fig. 17b of Hewett \& Madnia (1998) from their Runs 1 and 5. While their simulations are of a somewhat different problem, comparing their Runs 3 and 5 in their Fig. 12c further supports the present observation that dilatation is the dominant effect of heat release in a reacting vortex ring.

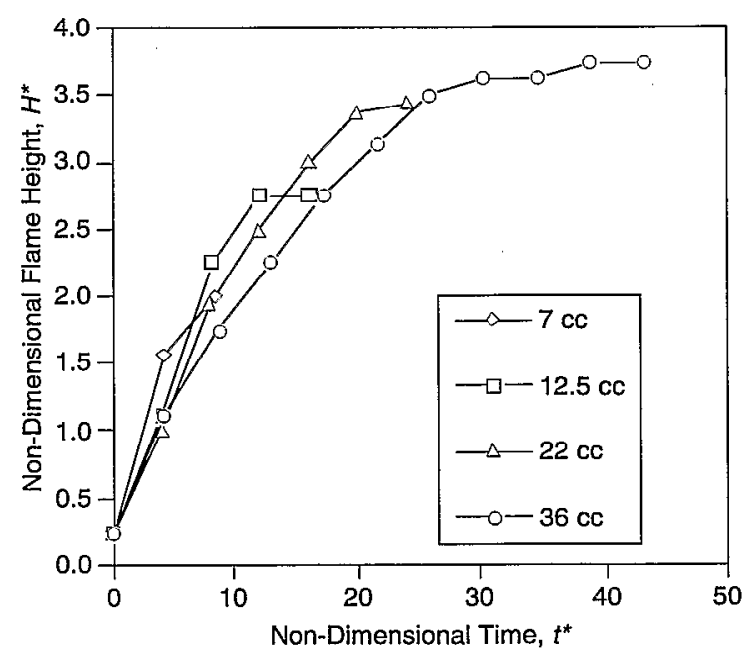

Figure 8. Methane-air experimental results for the effect of fiuel volume $V_{F}$ on vortex ring trajectory $H^{*}\left(t^{*}\right) . \Gamma=500 \mathrm{~cm}^{2} / \mathrm{s}$ for all cases. 
$t^{*}=2.5$
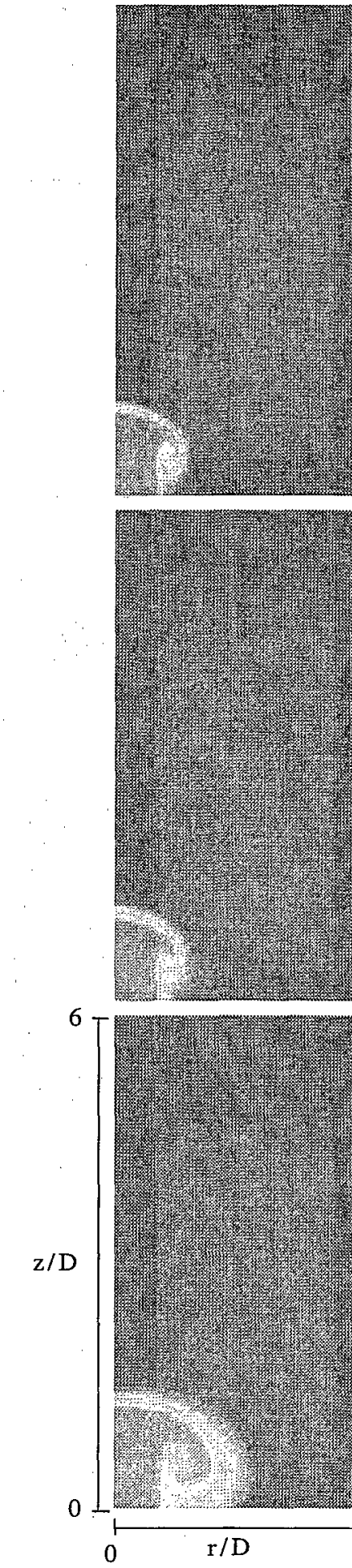

$t^{*}=6.25$
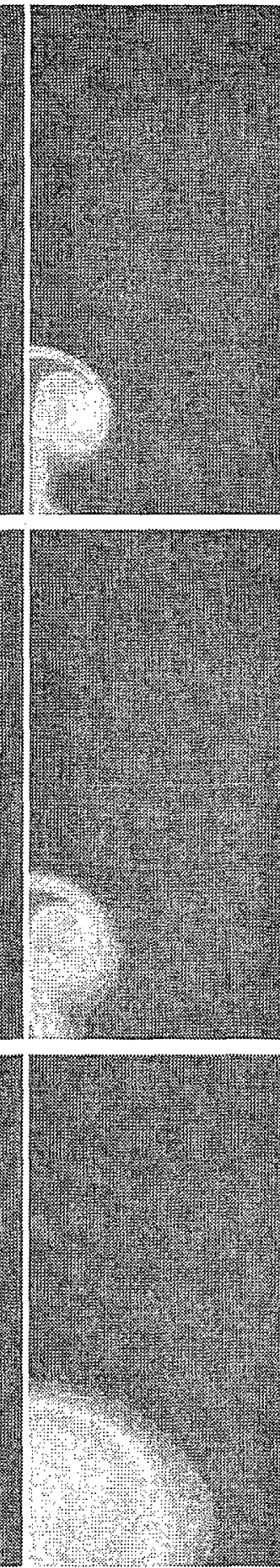

$t^{*}=12.5$
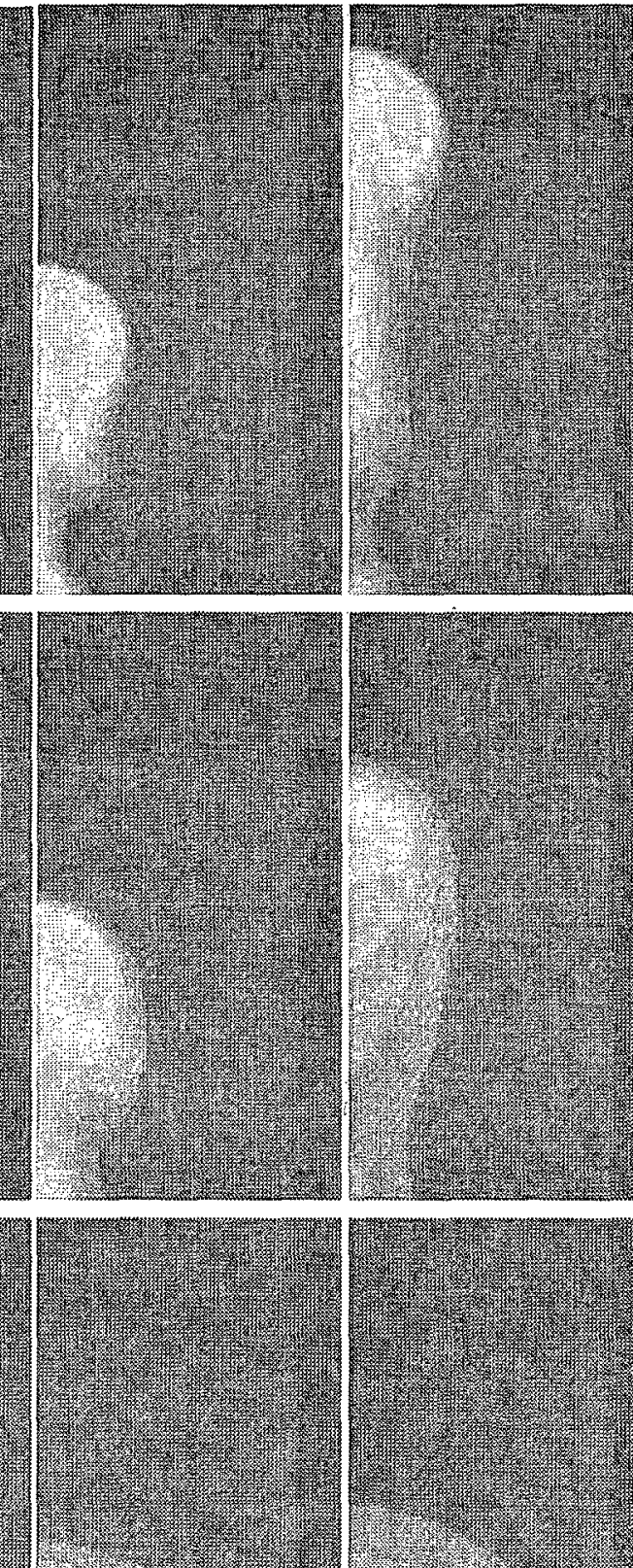


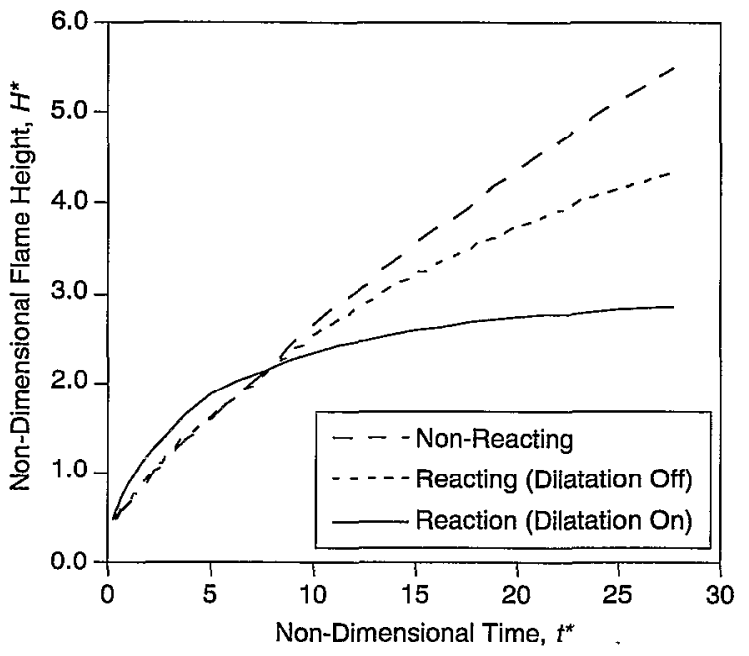

Figure 10. Comparison of methane-air simulation results for combustion release effects on vortex ring trajectory $H^{*}\left(t^{*}\right) . \quad V_{F}=10.5 \mathrm{cc}, \Gamma=500$ $\mathrm{cm}^{2} / \mathrm{s}$, and $T_{\max }=2226 \mathrm{~K}$ in all cases.

Besides the reduction in ring speed with time for any given case in Fig. 8, there are differences in the trajectories that are not accounted for by the hydrodynamic scaling $H^{*}\left(t^{*}\right)$ alone. The effect of fucl volume can also have an impact on both the structure and dynamics of reacting vortex rings. This effect is also found to be associated with dilatation and is explained next.

\subsection{Effect of Fuel Volume}

The structural and dynamical change in a reacting vortex ring due to fuel volume changes is seen in Fig. 5. In these experiments (Figs. 5a-c), the ring circulation is essentially kept constant, and by just varying the fuel volume the flame structure and dynamics are affected considerably. In Fig. 5a, a distinctive vortex ring shape is observed only in the early time. The later stage is more suggestive of a diffusion-limited burnout of the rich section on the centerline of the vortex ring. The increase of fuel volume in Figs. $5 \mathrm{~b}$ and $5 \mathrm{c}$ leads to the formation of a luminous bubble, and a ringlike structure can be seen in the burnout stage of Fig. 5c. During the early stage of Figs. $5 b$ and $5 c$, there are no major differences in the ring speed, however in the later stage, a large difference in ring speed can be observed because of a larger fuel volume in Fig. 5c.

An increase in fuel volume beyond the overfilled limit as mentioned above can lead to the formation of overfilled vortex ring, which displays azimuthal instability and formation of secondary and tertiary rings as seen in Fig. 5d. For the case of non-overfilled rings, the volume of fluid that rolled up during the ring formation from the $2-\mathrm{cm}$ diameter nozzle is approximately $50 \mathrm{~cm}^{3}$.

From the experimental results of methane-air reaction shown in Fig. 8 , it is evident that fuel volume also plays an important role in the ring dynamics. With the ring circulation and all other parameters essentially identical except for the fuel volume, one would expect the trajectories to look similar, yet there is a clear trend with fuel volume that suggests a further heat release effect.

The combustion in a reacting vortex ring can be approximated as

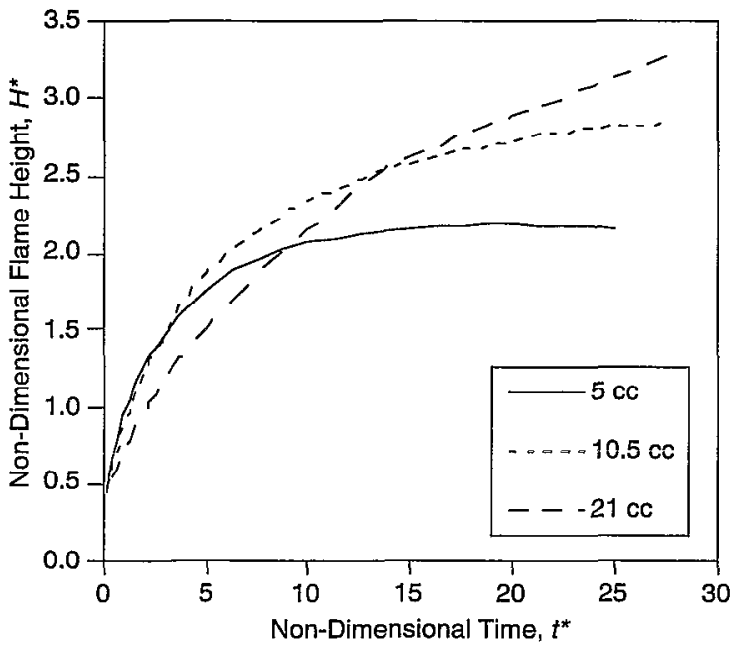

Figure 11. Methane-air simulation results showing effect of fuel volume $V_{F}$ on vortex ring trajectory $H^{*}\left(t^{*}\right) . \quad \Gamma=100 \mathrm{~cm}^{2} / \mathrm{s}$, and $T_{\max }=2226 \mathrm{~K}$ in all cases.

two phases. This has been suggested by experimental studies of Southerland et al (1991). The first is where the fuel and oxidizer, which initially roll up together during the ring formation phase, burn rapidly to consume the lcan reactant. The sccond phase is where any remaining fuel is consumed by the diffusion of the required oxidizer into the ring. An increase in fuel volume during the initial phase will decrease the amount of oxidizer that rolled up into the vortex ring. Depending on the stoichiometric requirements, there is a critical ratio of $V_{F} / V_{R}$ for which the oxidizer becomes the lean reactant. Beyond such limit, an increase in fuel volume will lead to less heat been released, which in turn, will lead to a decrease in ring speed in the early stage. For methane-air reaction the amount of fuel beyond which the oxidizer becomes the lean reactant is when $V_{F}=5$ $\mathrm{cm}^{3}$. This is based on the $50 \mathrm{~cm}^{3}$ of fluid that is rolled up during the ring formation for a $2-\mathrm{cm}$ diameter nozzle. This effect is clearly demonstrated in the experimental results of methane-air combustion in Fig. 8 where all cases exceed the stated critical fuel volume.

A numerical simulation was also conducted to address the effect of fuel volume on the reacting ring dynamics. Figure 11 shows the effect of fuel volume on the ring trajectories of methane-air reactions for fuel volumes ranging from 5 to $21 \mathrm{cc}$. Consistent with the experimental results of Fig. 8, the numerical results do show that increasing fuel volume beyond the critical limit will lead to a reduction in ring speed in the early stage. On the other hand, in the later stage the increase in fuel volume contributed to an increase in ring heights, and this is expected since there is more fuel to be consumed in the larger fuel volume case.

\subsection{Effect of Ring Circulation}

In a nonreacting vortex ring, an increase in ring circulation leads to an increase in ring speed, but when plotted in hydrodynamically scaled variables all the trajectories should collapse to a single curve, assuming similar core structures, since the ring circulation merely scales the characteristic time. While the scaled trajectories will be independent of circulation, an increase in ring circulation can also increase mixedness of fuel 


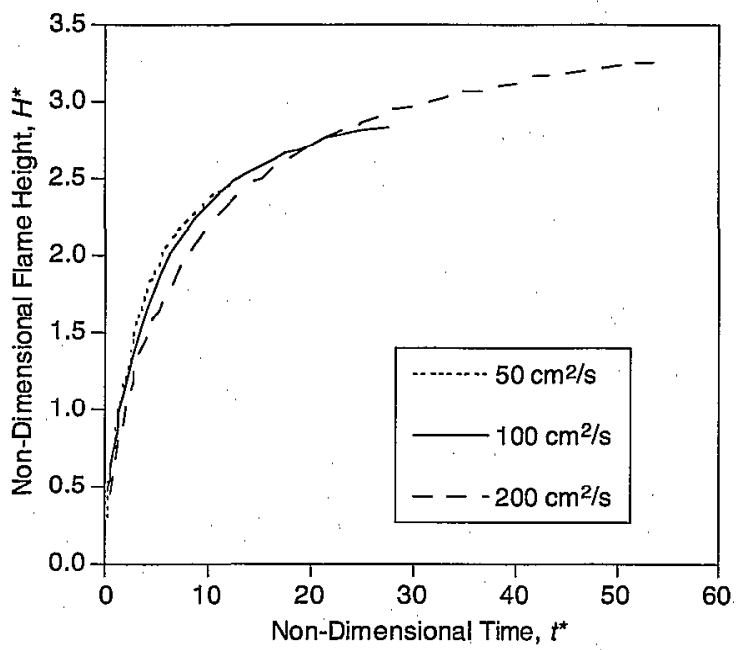

Figure 12. Methane-air simulation results showing effect of ring circulation $\Gamma$ on vortex ring trajectory $H^{*}\left(t^{*}\right), V_{F}=10.5 \mathrm{cc}$, and $T_{\max }=$ $2226 \mathrm{~K}$ in all cases.

and oxidizer inside a vortex ring (Verzicco \& Aguirre, 1995), and result in the thinning of the diffusion layer on the forward stagnation point of the vortex ring due to an increase in strain rate. However, as noted above, in a reacting vortex ring dilatation due to combustion heat release affects the ring trajectory. With the increase in mixedness, one should expect to have increased dilatation for larger ring circulations.

A numerical study was conducted to determine the effect of ring circulation on the reacting vortex rings. Figure 12 shows the numerical results of three ring circulation cases with time and height hydrodynamically scaled, and to a first order approximation, the trajectories do collapse to a single curve. However, the small differences in ring speed seen in $t^{*}<20$ indicate that an increase in ring circulation contributes to a decrease in ring speed. This is unexpected since more mixedness from an increase in ring circulation should increase dilatation. For a fixed fuel volume, the increase in ring circulation is achieved by reducing the total fuel injection time. The higher rate of fuel injection in the higher ring circulation case is similar to the effect of fuel volume as discussed above where higher fuel volume leads to a reduction in dilatation. As figure 12 indicates, the lower ring circulation does show the result of higher dilatation as compared to the higher circulation cases, during the early phase of the interaction. A numerical study was also conducted to determine whether fuel volume plays a role. It was found that even for fuel volumes below the critical value (5 $c c)$, the same trend was seen and this further suggests that the rate of fuel injection is also of importance.

From these numerical studies, it is found that enhanced mixedness due to an increase in ring circulation as observed in nonreacting vortex ring appears not to contribute to an increase in dilatation for the reacting vortex rings. Moreover, the small differences seen in Fig. 12 are attributable to the change in the rate of fuel injection resulting from a change in ring circulation.

\subsection{Effect of Stoichiometry}

The comparison of Figs. 5c and 6 allows the effect of

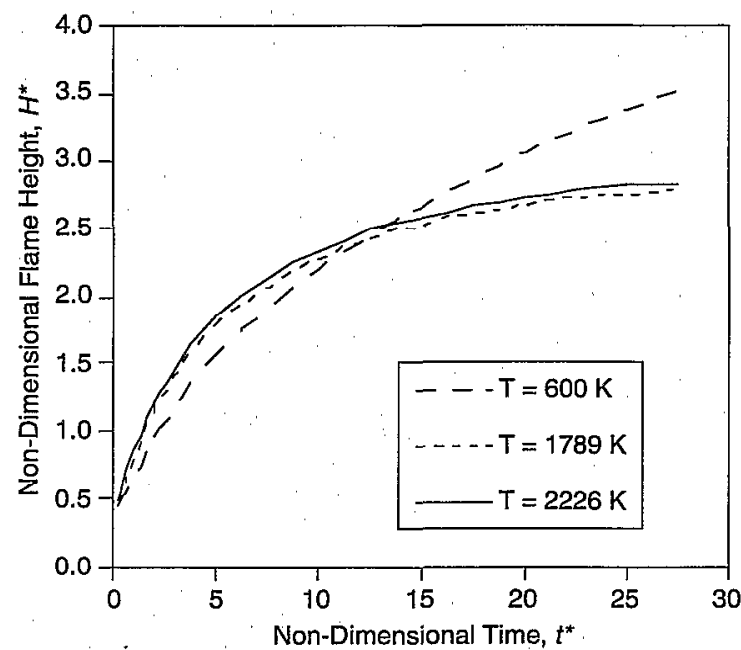

Figure 13. Methane-air simulation results showing effect of $T_{\max }$ on vortex ring trajectory $H^{*}\left(t^{*}\right) . V_{F}=10.5 \mathrm{cc}$ and $\Gamma=100 \mathrm{~cm}^{2} / \mathrm{s}$ in all cases.

stoichiometry on the reacting vortex rings to be examined by varying the nitrogen dilution of the fuel. In the three cases, the ring circulation and fuel volume are kept essentially constant. Nitrogen dilution of the fuel has the effect 'of moving the stoichiometric surface closer to the fuel side with little change in adiabatic flame temperature. For a given strained laminar flame with thickness $\lambda_{D}$ the separation between the stoichiometric surface and the nominal air-fuel interface is given by $n_{s} / \lambda_{D}=$ $e r f^{-1}(1-\phi) /(1+\phi)$.

The flame structure in all three cases looked somewhat similar, and this is consistent with the results in Fig. 12 of Manda and Karagozian (1988) for their range of $f^{*}$ between 0.1 and 0.35 . The dominant effects of nitrogen dilution of the fuel appear to be a reduction in flame luminosity and flame burnout time. Figure $4 \mathrm{c}$ of the pure propane case shows a luminous region that envelops the whole ring and burns out from both the leading and trailing ends. In the case of $60 \%$ nitrogen with $40 \%$ propane in Fig. 6a, a bright luminous cap is present only very near the leading edge of the flame and dissipates toward the leading edge. As for the case of $20 \%$ propane with $80 \%$ nitrogen in Fig $6 \mathrm{~b}$, no bright luminous cap is formed, however, the brightest region is still located at the leading edge. The reduction in flame burnout time as nitrogen dilution increases is most likely connected to less soot been available for the soot burnoul phase which cannot be differentiated from the recorded luminosity.

\subsection{Effect of Radiative Heat Loss}

Numerical results of Fig. 13 and the experimental results of Figs. 14 and 15 were used to determine the effect of radiative heat loss on reacting vortex rings. Comparison of Figs. 14 and 15 suggests marked differences in both the burnout time and flame structure even though both cases have similar ring circulation and fuel volume, except the fuel type. One explanation is the increased in radiative heat loss in Fig. 15 due to the higher sooting propensity of propane which may be responsible for the difference in flame shapes. The formation of the luminous cap proceeds in a similar way, but the cap dissipates in a very differently in the late stage of interaction. 


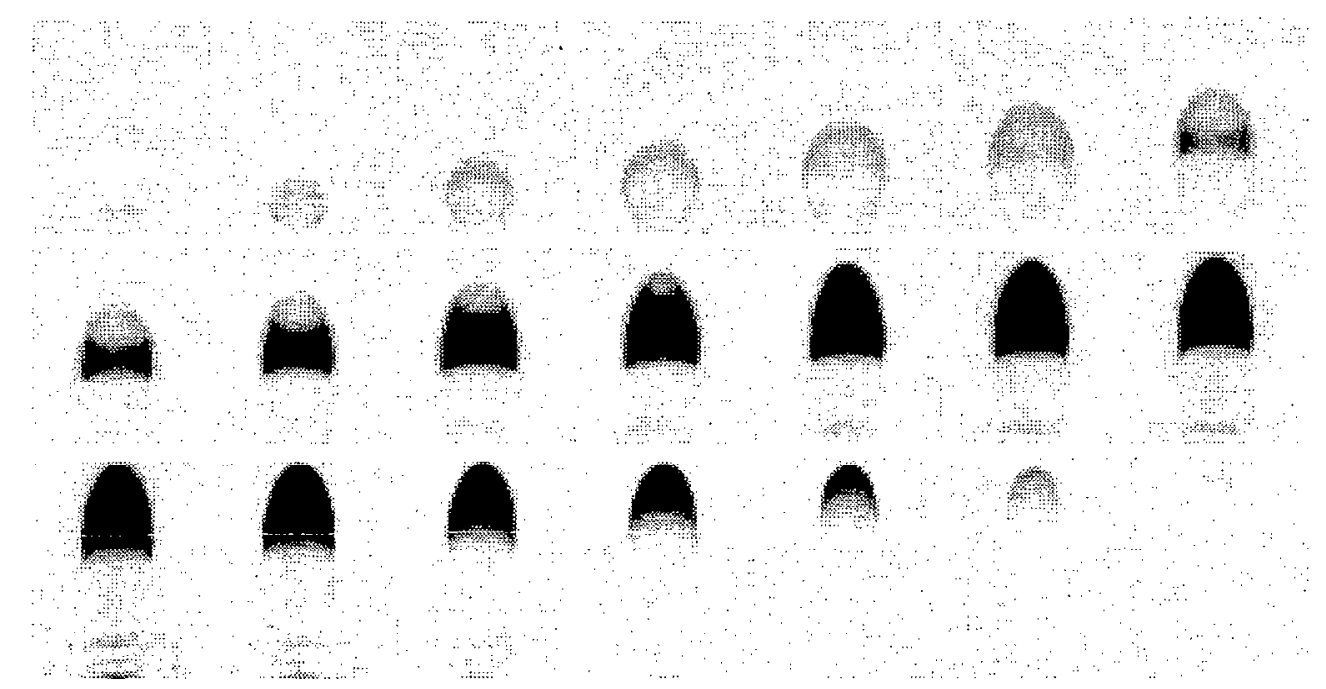

Figure 14. Flame-vortex interaction for ethane burning in air, $\Gamma=282 \mathrm{~cm}^{2} / \mathrm{s}$ and $V_{F}=26 \mathrm{cc}$. Flame burnout time is $667 \mathrm{~ms}$.

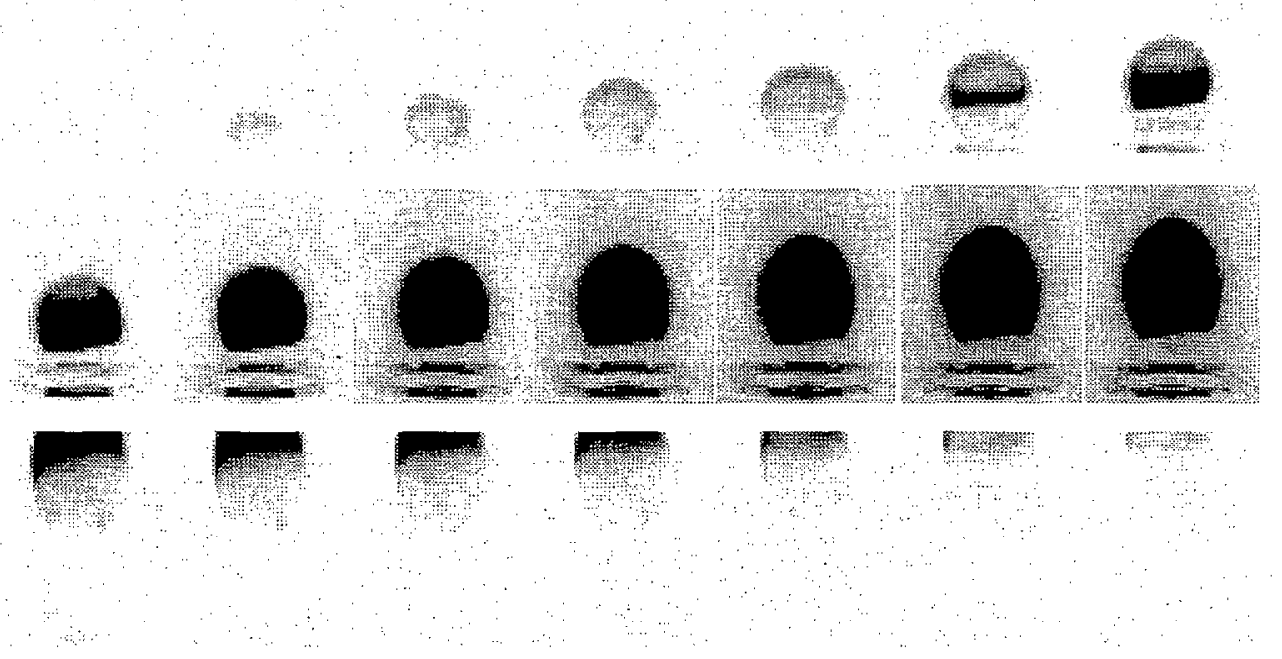

Figure 15. Flame-vortex interaction for propane burning in air, $\Gamma=273 \mathrm{~cm}^{2} / \mathrm{s}$ and $V_{F}=26 \mathrm{cc}$. Flame burnout time is $1233 \mathrm{~ms}$.

Since dilatation is the dominant heat release effect in this configuration, numerical results of Fig. 13 determine whether the difference in flame shapes in Figs. 14 and 15 may be due to reduced dilatation from a difference in the radiative heat loss. In the numerical study, radiative heat loss is simply modeled as a reduction in $T(\zeta)$, however, Fig. 13 shows that for a reasonable reduction in $T_{\max }$ the effect on the ring trajectory is minimal. This suggests that while dilatation suffices to explain most of the heat release effects discussed here, other mechanisms may be responsible for the differences seen in Figs. 14 and 15.

\section{CONCLUSIONS}

Flame-vortex interactions provide a mean for studying many of the fundamental aspects on the coupling between flow and reaction found in turbulent reacting flows. The combined efforts of experimental and numerical studies have demonstrated that dilatation is the dominant effect of combustion heat release; this is also consistent with the numerical simulations of Hewett \& Madnia (1998) of a somewhat different configuration.

Fuel volumes play a role in determining the amount of heat release during the initial stage, and it was found that increasing fuel volume beyond a critical limit as determined by stoichiometric requirements, while keeping the circulation constant, can result in a reduction in heat release which in turn leads to a decrease in ring speed. Experimental results of pure propane fuel showed a clear effect of the fuel volume on the flame structure and burnout time for rings that do not exceed the overfilled limit.

The increase in ring circulation with fuel volume kept constant gives a reduction in ring speed during the early stage of 
interaction. Results from nonreacting vortex rings hinted to an increase in dilatation from an enhanced mixedness due increasing circulation. However, the numerical results for reacting vortex rings showed that enhanced mixedness has a minimal effect on dilatation for the range of ring circulation studied here.

The effect of nitrogen dilution of propane cases was a major reduction in flame luminosity and burnout time. However the flame shape was not considerably affected by the nitrogen dilution, and this was in agreement with the analytical results of Karagozian \& Manda (1986) and Manda \& Karagozian (1988) for their vortex pair case. The reduction in burnout time is most likely due to the presence of less soot as nitrogen dilution is increased.

Experimental results revealed that radiative heat loss can affect the flame structure and dynamics in a reacting vortex ring. Numerical studies that mimic radiative heat loss by lowering the adiabatic flame temperature did not reproduce the differences seen between the experimental results of propane and ethane cases. This suggests that a mechanism other than the simple reduction in dilatation due to radiative heat loss is responsible for the marked differences.

\section{Acknowledgements}

This work is supported by the NASA Microgravity Combustion Science Program under Contract Nos. NASA-G-NCC3-663 and NASA-G-NAG3-1639.

\section{References}

Baum, H.R., Rehm, R.G., and Gore, J.P. (1990) Transient combustion in a turbulent eddy, Proc. 23 rd Symp. (Int'l) Comb., The Combustion Institute, Pittsburgh, PA, 715-722.

Chen, L.D., Roquemore, W.M., Goss, L.P., and Vilimpoc, V. (1991) Vorticity generation in jet diffusion flames, Comb. Sci. Tech. $77,41-57$.

Chen, S.-J., and Dahm, W.J.A. (1997) Vortex ring/diffusion flame interactions in microgravity conditions, Fourth International Microgravity Combustion Workshop, NASA CP10191, 191-196.

Chen, S.-J., and Dahm, W.J.A. (1998) Diffusion flame structure of laminar vortex ring under microgravity conditions, Proc. 27th Symp. (Int'l) Comb., The Combustion Institute, Pittsburgh, PA, 2579-2586.

Chen, S.-J., and Dahm, W.J.A. (1999a) Experiments on diffusion flame structure of a laminar vortex ring, First Joint Meeting of the U.S. Sections of the Combustion Institute, The Combustion Institute, Pittsburgh, PA, 461-464.

Chen, S.-J., and Dahm, W.J.A. (1999b) The interaction of a vortex ring, with a diffusion flame under microgravity conditions, Fifth International Microgravity Combustion Workshop, NASA CP-208917, 271-274.

Gharib, M., Rambod, E., and Shariff, K. (1998) A universal time scale for vortex ring formation, J. Fluid Mech., 360, 121140 .

Hewett, J.S., and Madnia, C.K. (1998) Flame-vortex interactions in a reacting vortex ring, Phys. Fluids, 10, 189-205.
Hsu, K.Y., Chen, L.D., Katta, V.R., Goss, L.P., and Roquemore, W.M. (1993) Experimental and numerical investigations of the vortex-flame interactions in a driven jet diffusion flame, AIAA PaperNo. 93-0455, AIAA, Washington, DC.

Karagozian, A.R., and Manda, B.V.S. (1986) Flame structure and fuel consumption in the field of a vortex pair, Comb. Sci. Tech., 49, 185-200.

Karagozian, A.R., Suganuma, Y., and Strom, B.D. (1988) Experimental studies in vortex pair motion coincident with a liquid reaction, Phys. Fluids, 31 (7), 1862-1871.

Manda, B.V.S., and Karagozian, A.R. (1988) Effects of heat release on diffusion flame-vortex pair interactions, Comb. Sci. Tech., 61, 101-119.

Marble, F.E. (1985) Growth of a diffusion flame in the field of a vortex, in Recent Advances in the Aerospace Sciences, (C. Casci, Ed.), Plenum Press, New York, NY, 395-413.

Park, J., and Shin, H.D. (1997) Experimental investigation of the developing process of an unsteady diffusion flame, Combustion and Flame, 110, 67-77.

Renard, P.H., Rolon, J.C., Thévenin, D., and Candel, S. (1999) Evolution of the flame structure for a nonpremixed flame interacting with a vortex, Comb. Flame, 117, 189-205.

Rolon, J.C., Aguerre, F., and Candel, S. (1995) Experiments on the interaction between a vortex and a strained diffusion flame, Comb. Flame, 100, 422-429.

Southerland, K.B., Porter III, J.R., Dahm, W.J.A., and Buch, K.A. (1991) An experimental study of the molecular mixing process in an axisymmetric laminar vortex ring, Phys. Fluids, A 3, 1385-1392.

Strawa, A.W., and Cantwell, B.J. (1985) Visualization of the structure of a pulsed methane-air diffusion flame, Phys. Fluids, $28(8), 2317-2320$.

Takahashi, F., Schmoll, W.J., Trump, D.D., and Goss, L.P. (1996) Vortex-flame interactions and extinction in turbulent jet diffusion flames, Proc. 26th Symp. (Int'l) Comb., 145-152.

Thévenin, D., Rolon, J.C., Renard, P.H., Kendrick, D.W., Veynante, D., and Candel, S. (1996) Structure of a nonpremixed flame interacting with counterrotating vortices, Proc. 26th Symp. (Int'l) Comb., The Combustion Institute, Pittsburgh, PA, 145-152.

Vandsburger, U., Seitzman, J.M., and Hanson, R.K. (1988) Visualization methods of the study of unsteady non-premixed jet flame structure, Comb. Sci. Tech., 59, 455-461.

Verzicco, R., and Orlandi, P. (1995) Mixedness in the formation of a vortex ring, Phys. Fluids, 7 (6), 1513-1515.

You, Y.H., Lee, D.K.,, and Shin, H.D. (1998) Visual investigation of a vortex ring interacting with a nonpremixed flame, Comb. Sci. Tech., 139, 365-383. 